\title{
WRIGHT TYPE MULTIPLICATIVELY CONVEX FUNCTIONS
}

\author{
KAIZHONG GUAN
}

Abstract. The notion of Wright type multiplicatively convex functions is introduced. Relationships between such functions and multiplicatively convex functions are investigated, and a counterpart of the $\mathrm{Ng}$ representation theorem for Wright convex functions is presented. It is proved that the function $F\left(x_{1}, \ldots, x_{n}\right)=f\left(x_{1}\right) \cdots f\left(x_{n}\right)$ is multiplicatively Schur-convex if and only if $f$ is Wright type multiplicatively convex. A Hermite-Hadamard type inequality for Wright type multiplicatively convex functions is also given.

Mathematics subject classification (2010): 26A51, 26D15.

Keywords and phrases: Convexity, multiplicative convexity, Wright-convex, Wright type multiplicative convexity, multiplicative Schur-convexity.

\section{REFERENCES}

[1] I. C. Draghicescu, Problem 2810, Crux Mathematicorum, 29 (1) (2003), 46.

[2] K. Z. GuAN, Multiplicative convexity and its applications, J. Math. Anal. Appl., 362 (2010), 156-166.

[3] Pl. Kannappan, Functional Equations and Inequalities with Applications, Springer, Dordrecht Heidelberg/Lodon/New York, 2009.

[4] P. Montel, Sur les fonctions convexes et les fonctions soushamoniques, Joutnal de Math., 9 (7) (1928), 29-60.

[5] C. T. NG, Functions generating Schur-convex sums, in: W. Walter (Ed.), General Inequalities, 5, Oberwolfach, 1986, in: Internat. Ser. Numer. Math., vol. 80, Birkhäuser, Basel, Boston, 1987, p. 433-438.

[6] K. Nikodem, On some class of midconvex functions, Ann. Pol. Math., 50 (1989), 145-151.

[7] C. P. Niculescu, Convexity according to the geometric mean, Math. Inequal. Appl., 2 (2000), 155167.

[8] C. P. Niculescu, L. E. Persson, Convex Functions and Their Applications, A Contemporary Approach, CMS Books in Mathematics, vol. 23, Springer-Verlag, New York, 2006.

[9] A. W. Roberts, D. E. Varberg, Convex Functions, Pure Appl. Math., vol. 57, Academic Press, New York, 1973.

[10] E. M. Wright, An inequality for convex functions, Amer. Math. Monthly, 61 (1954), 620-622.

[11] X. M. Zhang, Geometrically-convex Functions (in Chinese), Anhui University Press, Hefei, 2004. 